COPYRIGHT @ 2018 INTERNATIONAL JOURNAL OF SCIENCE DENTISTRY | AVAILABLE ONLINE

http://www.periodicos.uff.br/index

\title{
REABILITAÇÃO EM PROTOCOLO ALL-ON-SIX : RELATO DE CASO \\ CLÍNICO
}

ALL-ON-SIX PROTOCOL REHABILITATION: CASE REPORT

\section{Cíntia Freire}

Aluna da graduação da Faculdade de Odontologia, Universidade Federal Fluminense UFF.

\section{Rafael Coutinho}

Aluno de Doutorado em Odontologia Universidade Federal Fluminense - UFF.

\section{Camila Diuana de Almeida}

Especialista em Prótese Dentária, Universidade Federal do Rio de Janeiro - UFRJ.

\section{Suelen Cristina Sartoretto}

Professora de Cirurgia Bucal e Anatomia da Faculdade de Odontologia da Universidade Iguaçu - UNIG.

\section{Monica Diuana Calasans-Maia}

Professora titular da disciplina de Cirurgia Oral Menor da Universidade Federal Fluminense - UFF.

Instituição: Universidade Federal Fluminense

Categoria: Relato de caso

Informações do autor principal: Cíntia Freire, Rua Barão do Amazonas nº. 280, apt 201-A, Centro, CEP 24030-110, Niterói, RJ. cintiafreiree@ hotmail.com 
COPYRIGHT @ 2018 INTERNATIONAL JOURNAL OF SCIENCE DENTISTRY | AVAILABLE ONLINE http://www.periodicos.uff.br/index

RESUMO:A técnica de reabilitação com protocolos buscando a redução do número de implantes instalados tem sido realizada com excelentes taxas de sobrevivência e sucesso, além de apresentar uma grande previsibilidade e precisão dos resultados. $\mathrm{O}$ objetivo deste relato de caso foi descrever um protocolo reabilitador pela técnica de redução do número de implantes, com prototipagem reversa em maxila. Paciente do sexo masculino, 59 anos de idade, apresentou-se com múltiplas raízes residuais superiores e com uma condição precária de higiene oral. Após a avaliação clínica e radiográfica do paciente foi sugerido a reabilitação em protocolo all-on-six. Foram realizadas extrações de 15 raízes residuais, moldagem total superior e inferior, tomografia computadorizada, confecção de guia cirúrgico, confecção da prótese total superior, instalação de 6 implantes dentários e reabilitação protética. Esta técnica permitiu a obtenção de resultados funcional e estético satisfatórios, de rápida execução, com custo reduzido e menor morbidade.

Palavras-chave: all-on-four; planejamento digital; Reabilitação protética.

ABSTRACT: The technique of rehabilitation with protocols looking for the reduction of the number of installed implants has been performed with excellent rates of survival and success, besides presenting a great predictability and precision of the results. The purpose of this case report was to describe a rehabilitation protocol with the reduction of the number of installed implants, with reverse prototyping of maxilla. A 59-year-old male patient presented with residual roots of 15 all upper teeth and with a precarious oral hygiene condition. After the clinical and radiographic evaluation it was suggested an all-on-six protocol. fifteen residual roots were extracted, upper and lower total molding, computed tomography, surgical guide, superior total prosthesis preparation, 6 dental implants and prosthetic rehabilitation. This technique allowed to obtain satisfactory functional and aesthetic results with fast execution, with reduced cost and lower morbidity.

Key words: All-on-four; Digital planning; Prosthetic rehabilitation.

REVISTA FLUMINENSE DE ODONTOLOGIA - ANO XIV - No 50 - Julho / Dezembro 2018 


\section{INTRODUÇÃO}

O conceito de osseointegração em implantes surgiu com Branemark, P.I., 1969 a partir dos anos de 1960 e outras técnicas evoluíram para aprimorar a previsibilidade desse conceito. Muitos estudos na área mostram a relevância em benefícios para a saúde de pessoas reabilitadas com implantes dentários, além da função estomatognática, benefícios estéticos, fonéticos e psicológicos alcançados (BRANEMARK, P.I., 1969).

Com o advento da Implantodontia, hoje consagrada pelas altas taxas de sucesso e sobrevivência, a osseointegração simples perdeu espaço para alguns outros conceitos mais avançados, como o posicionamento tridimensional dos implantes, a relação entre diâmetro e altura dos implantes dentários, o nível de inserção dos implantes instalados e tempo de reabilitação (PUIG, C.P., 2010).

O uso de prótese total fixa com carga imediata ou precoce hoje representa uma realidade no tratamento de pacientes completamente edêntulos com altas taxas de sucesso na maxila (93\% a 99,2\% com de 5 anos de acompanhamento) e mandíbula (93,2\% a 100\% com 5 anos de acompanhamento) (VIEIRA, R.A., 2014).

A prótese total fixa aparafusada sobre quatro (All-on-four) à seis (All-on-Six) implantes teve seu início revolucionando reabilitações de maxila e mandíbula edêntulas completas, o conceito surgiu para interpor a ideia de quanto mais implantes a prótese torna-se mais estável sem levar em consideração o posicionamento dos implantes e a biomecânica necessária nas reabilitações de arcos totais, que até então era o preconizado pelos estudos da área (BRANEMARK, P.I.,1969). Maló, P., 2011 relatou que, seguindo critérios clínicos e exames de imagem, pode-se planejar um caso de edentulismo total superior com 4 à 6 implantes e inferior com 4 implantes com sucesso e perfeita osseointegração, até mesmo sendo possível fazer a instalação dessas próteses totais em um único estagio cirúrgico ou em carga imediata (MALÓ, P., 2017; MOURÃO, C., 2017).

Para a execução do protocolo sugerido por Maló. P., 2011 devemos respeitar algumas diretrizes importantes como, torques superiores a $30 \mathrm{Ncm}$ e preferencialmente implantes de altura suficiente para ancoragem em fossa canina. Dessa forma julga-se 
COPYRIGHT @ 2018 INTERNATIONAL JOURNAL OF SCIENCE DENTISTRY | AVAILABLE ONLINE http://www.periodicos.uff.br/index

obter uma distribuição melhor dos implantes, minimizando os problemas de osteointegração causada pela extensão dos cantileveres distais (MALÓ, P., 2011).

O conceito de tratamento "all-on-four" foi desenvolvido para maximizar o uso do osso remanescente disponível nas mandíbulas atróficas, permitindo a função imediata e evitando procedimentos regenerativos que aumentam os custos do tratamento e a morbidade do paciente, bem como as complicações inerentes a esses procedimentos.

Com a evolução dos exames complementares e da Odontologia Digital novas ferramentas foram introduzidas a fim de melhorar e facilitar os procedimentos cirúrgicos sobre implantes. A tomografia computadorizada (TC) exame de imagem que surge nesse panorama como uma grande aliada, assim como os arquivos STereoLithography (STL), que permite o planejamento tridimensional com a ajuda de um software, assim sendo possível avaliar a densidade óssea, viabilizar a cirurgia dos casos virtualmente planejados e entender o tratamento do pacientes anteriormente ao procedimento cirúrgico (planejamento reverso). Com a cirurgia guiada pode-se definir previamente a quantidade de implantes, o local de instalação e as dimensões dos implantes. Dessa forma, escolher qual técnica é a mais indicada e qual a melhor opção protética na reabilitação de casos específicos(MOURÃO, C., 2017).

Outro benefício é a confecção do guia cirúrgico a partir da TC, que permite que os implantes sejam instalados com o orifício e posição de acesso para o implante predeterminado pelo software, o que permite uma maior previsibilidade e precisão dos resultados (VAN ASSCHE, N., 2012).

A qualidade de vida e o conforto da reabilitação com implantes é muito superior quando comparada à uma prótese total mucossuportada, oferecendo uma cirurgia minimamente invasiva, previsível e com resultados promissores, o que permite que esse paciente seja novamente integrado à sociedade, promovendo melhora na saúde oral e qualidade de vida.

Nesse relato de caso clínico será apresentado a utilização de novas tecnologias a partir de um guia cirúrgico implanto suportado como alternativa de tratamento para pacientes edêntulos totais, auxiliando a melhor previsibilidade em tratamentos com um número de implantes reduzidos.

REVISTA FLUMINENSE DE ODONTOLOGIA - ANO XIV - No 50 - Julho / Dezembro 2018 
COPYRIGHT @ 2018 INTERNATIONAL JOURNAL OF SCIENCE DENTISTRY | AVAILABLE ONLINE http://www.periodicos.uff.br/index

\section{CONSIDERAÇÕES ÉTICAS}

Os procedimentos empregados seguiram os padrões éticos da Declaração de Helsinque de 1975, revisada em 2000. O paciente foi submetido a tratamento cirúrgico na Universidade Federal Fluminense, Rio de Janeiro, Brasil, após assinar o termo de consentimento livre e esclarecido para uso de imagens e dados para publicação científica. O estudo foi conduzido de acordo com as Diretrizes do Relatório de Caso Clínico (CARE, www.care-statement.org).

\section{RELATO DE CASO}

Paciente sexo masculino, 59 anos, leucoderma, não tabagista, queixava-se das ausências dentárias causadas por acidente. No pré-tratamento foi realizada a anamnese do paciente, fotos iniciais, exames por imagem através de radiografia panorâmica dos maxilares (Figura 1A) e moldagem em alginato para a confecção dos modelos de estudo. Durante o exame clínico, não foram observadas contraindicações locais ou sistêmicas para realizar o procedimento sob anestesia local em ambiente ambulatorial. Foi realizado o risco cirúrgico por seu médico e o parecer foi de ASA II, de acordo com a avaliação física proposta pela Sociedade Americana de Anestesiologistas em 1962. Exames de imagens foram realizados para quantificar o osso no sentido ântero-posterior e vestíbulo-lingual e a possível proximidade de estruturas anatômicas nobres, como o seio maxilar e fossa nasal visando evitar complicações transoperatórias. A avaliação constatou importantes perdas dentárias, doença periodontal e muitas raízes residuais (Figura 1B e 1C), que definiram o planejamento com a realização de um protocolo híbrido em resina com instalação média de 4 a 6 implantes conforme a necessidade de distribuição biomecânica dos implantes.

Todas as raízes residuais superiores foram extraídas em sessão única (Figura 1D). Para isso, foi administrada anestesia local com lidocaína a $2 \%$ epinefrina 1: 100.000 (Alphacaíne®, Nova DFL, Rio de Janeiro, RJ, Brasil), seguida das exodontias múltiplas. Com a pinça Beyer Biarticulada (Golgran, São Caetano do Sul, São Paulo, Brasil) foi realizada a regularização óssea. Após 30 dias (Figura 1E) o processo de cicatrização estava completo e permitiu a moldagem de estudo a fim de iniciar o

REVISTA FLUMINENSE DE ODONTOLOGIA - ANO XIV - No 50 - Julho / Dezembro 2018 
COPYRIGHT @ 2018 INTERNATIONAL JOURNAL OF SCIENCE DENTISTRY | AVAILABLE ONLINE http://www.periodicos.uff.br/index

processo de planejamento reverso, dando início ao planejamento cirúrgico-protético do paciente, respeitando os parâmetros estomatognáticos.
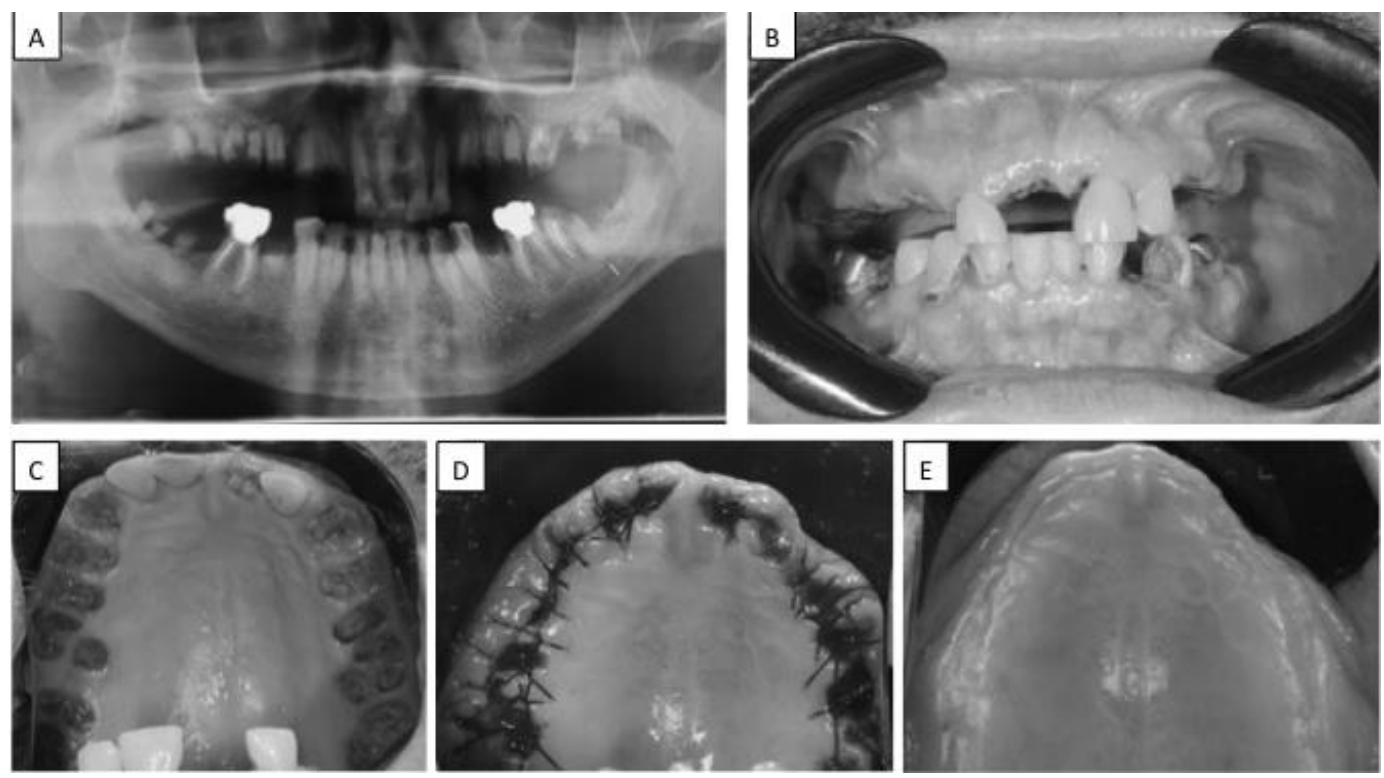

Figura 1. (A) Radiografia panorâmica inicial; (B e C) imagens clínicas intra-orais iniciais frontal e oclusal superior do paciente com presença de múltiplas raízes residuais; (D) sutura simples interrompida após extração seriada dentes superiores; (E) aspecto intra-oral do rebordo cicatrizado no pós-operatório.

Foi planejado como plano de tratamento inicial um protocolo superior do tipo All-onfour podendo ser finalizado com 4 ou 6 implantes respeitando a estabilidade inicial no momento da instalação dos implantes. Na etapa cirúrgica o planejamento cirúrgico virtualmente guiado por dupla tomografia foi realizado seguindo o POP (Procedimento Operacional Padrão) do planejamento reverso. Inicialmente foi realizada análise clínica da flacidez da mucosa da crista alveolar, seguida de moldagem utilizando material com características hidrofílicas para obtenção dos modelos. Logo após, uma moldeira de resina acrílica individual com vedação periférica para se obter um melhor fluxo de material durante a moldagem foi confeccionada, com isso, evita-se qualquer alteração ou pressão na mucosa alveolar. Após a criação de um modelo de trabalho, a confecção da placa base e rodete de cera de modelagem odontológica é conduzida para determinar a marcação do posicionamento tridimensional dos elementos dentários nesta prótese. $\mathrm{Na}$ concepção da prótese dentária, a flange anterior é removida na região da pré-maxila REVISTA FLUMINENSE DE ODONTOLOGIA - ANO XIV - No 50 - Julho / Dezembro 2018 
COPYRIGHT @ 2018 INTERNATIONAL JOURNAL OF SCIENCE DENTISTRY | AVAILABLE ONLINE http://www.periodicos.uff.br/index

para que seja possível verificar um melhor posicionamento da musculatura estomatognática. Neste momento foi possível diagnosticar o melhor tipo de prótese de acordo com o perfil labial do paciente. Em seguida foi realizada a montagem dos dentes alinhando as arcadas dentárias com posterior revisão do posicionamento muscular e após toda a revisão de posicionamento, iniciou-se a fase de duplicação desta prótese para a preparação do aparelho multifuncional incolor para mostrar possíveis áreas de pressão na mucosa.

Com o guia Multifuncional pronto foi realizado um exame clínico de adaptação do guia com posterior preparação desse mesmo guia para realização da tomada de referências para o planejamento cirúrgico virtual, onde acrescentados nessa moldeira 7 pontos aleatórios e não alinhados afim e facilitar o reconhecimento do software (coDiagnostiX ${ }^{\circledR}$, Dental wings, Canada) que planejou o posicionamento ideal dos implantes dentários. Com o guia multifuncional adaptado o paciente foi enviado ao centro radiológico (Data-X $®$, Barra da Tijuca, Rio de Janeiro) para realização de uma tomografia computadorizada do tipo Cone Beam (Icat@, Kavo, Biberach, Alemanha), onde inicialmente foi realizado uma tomografia do paciente com o guia multifuncional em posição e posteriormente somente a tomografia do guia sozinho. Com as duas tomografias realizadas foram encaminhadas ao centro de planejamento e confecção de guia cirúrgico virtualmente planejado (SmartSolution®, Rio de Janeiro, Brasil) (Figura 2A e 2B).Para o planejamento do posicionamento dos implantes dentários, inicialmente foi confeccionada uma guia cirúrgica com anilhas (muco-suportada) para instalação de 4 implantes, porém foi solicitado acrescentar mais 2 anilhas caso a instalação dos implantes mais posteriores e angulados não conseguissem uma ancoragem acima da estabilidade primária ideal (acima de $32 \mathrm{Ncm}$ ) (Figura 2C).

REVISTA FLUMINENSE DE ODONTOLOGIA - ANO XIV - No 50 - Julho / Dezembro 2018 


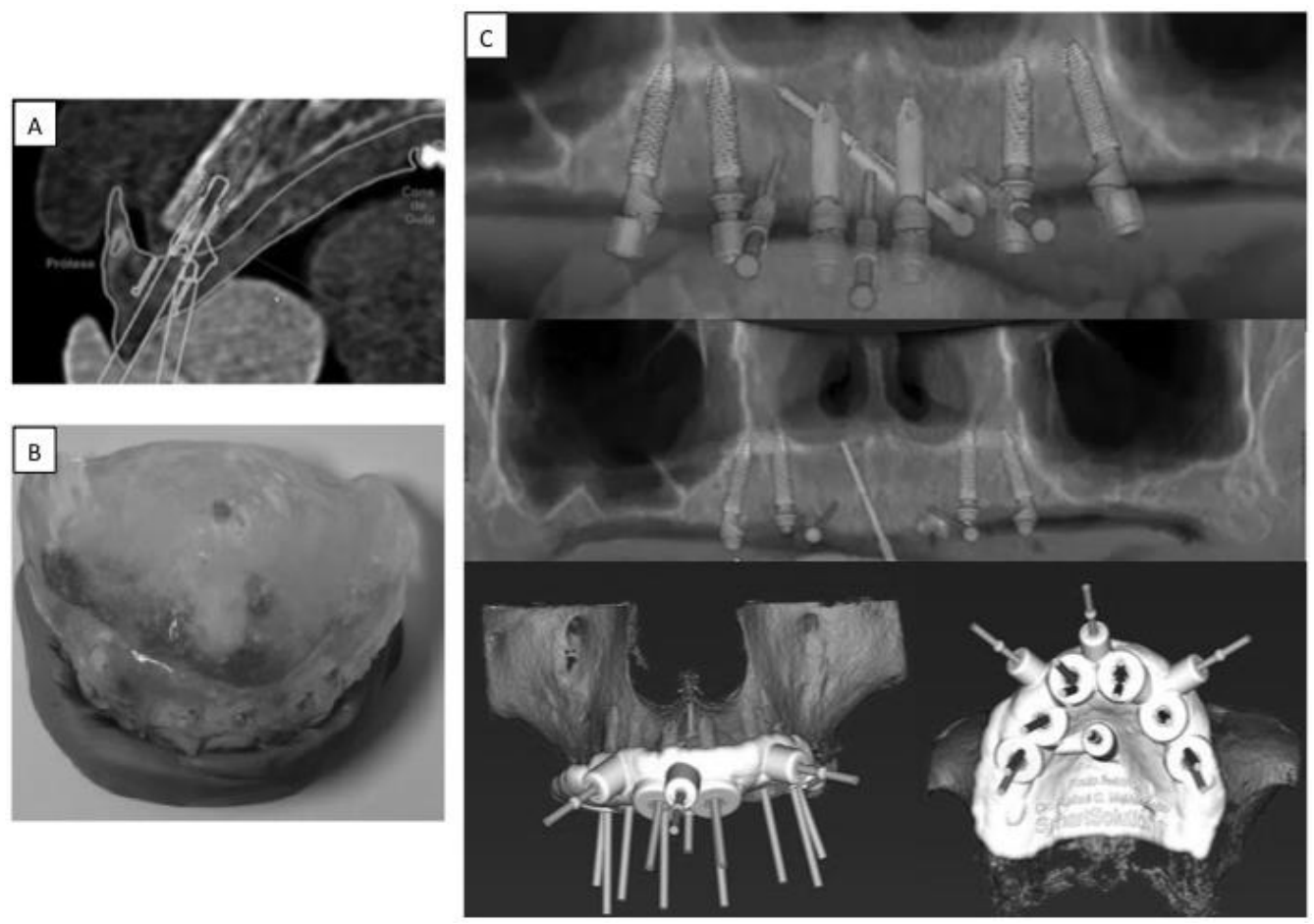

Figura 2.(A) Imagem tomográfica exemplificando o posicionamento do guia multifuncional na etapa de planejamento virtual dos Implantes;(B) guia multifuncional com guta percha para posicionamento e orientação dos implantes na tomografia; (C) imagem tomográfica apresentando o posicionamento de 6 implantes caso os implantes posteriores apresentem baixa estabilidade; abaixo, imagem do planejamento com 4 implantes e, abaixo, planejamento dos 6 implantes em guia cirúrgico.

Foi planejado, caso necessário, a instalação de até 6 implantes dentários do tipo Morse (EMFILS®, Itu, São Paulo), com medidas nos elementos 11 e 21 implantes de 3,5 x 13 mm, com Mini pilares (EMFILS®, Itu, São Paulo) com transmucoso de 1,5 de altura e com angulação de 17 graus. Nas regiões dos elementos 13 e 23, implantes de 3,5 x 13 mm, com Mini pilares (EMFILS®, Itu, São Paulo) com transmucoso de 1,5 de altura sem necessidade de angulação e nas regiões dos elementos 15 e 25, implantes de 3,5 x 13 mm, com Mini pilares (EMFILS®, Itu, São Paulo) com transmucoso de 1,5 de altura com angulação de 30 graus. Após o posicionamento da guia cirúrgica anilhada (Figura 3A) iniciou-se a fase de instalação dos implantes dentários utilizando o Kit Cirúrgico EmfilsGuide (EMFILS ®, Itu, São Paulo) (Figura 3D). Com a guia em 
COPYRIGHT @ 2018 INTERNATIONAL JOURNAL OF SCIENCE DENTISTRY | AVAILABLE ONLINE http://www.periodicos.uff.br/index

posição mucossuportada, inicialmente, foi realizada a estabilização da mesma pelos pinos de estabilização lateral (Figura 3B) que inicialmente foi feito uma perfuração com broca helicoidal de $1.3 \mathrm{~mm}$, em 4 pontos distintos.

$\mathrm{Na}$ fase inicial de perfuração para instalação dos implantes foi utilizado como guia, junto as anilhas, os guias de Brocas Estreito (utilizado para implantes de 3.5 de diâmetro) e para a instrumentação foram utilizadas 800 RPM com torque de 20 NCM com contra-angulo de 20:1.Progressivamente foi utilizada a broca lança 2.0 Estreito seguida das guias de brocas 2.5/2.8 Estreito junto com as respectivas brocas. Após a fase de preparo do leito ósseo, todos os implantes foram instalados utilizando inicialmente as chaves montadoras estreito para contra-ângulo com redução de 20:1 com as referências de 15 RPM com torque de 20 NCM (Figura 3C) Otorquímetro foi usado para precisão final da estabilidade primaria dos implantes, sendo os elementos 11 e 21 de $45 \mathrm{Ncm}$, os elementos 13 e 23 de $35 \mathrm{Ncm}$ e os elementos 15 e 25 de $32 \mathrm{Ncm}$.

Após a instalação final dos implantes (Figura 3E), no mesmo ato cirúrgico foi realizada a instalação de respectivos Minipilares com torque médio de $25 \mathrm{Ncm}$ já delimitado no relatório de planejamento enviado junto com o guia cirúrgico anilhado. Com a instalação dos minipilares, a fase de união foi iniciada usando um transferidor de mini-pilar de moldeira aberta, porém utilizando parafuso M2 (EMFILS®, Itu, São Paulo), na intenção de não ter interferência na oclusão após a união entre o guia Multifuncional e os cilindros de transferência. Após a instalação dos cilindros, os mesmos foram unidos a guia com auxílio de uma resina tipo Pattern (Resinlay®, TDV, Santa Catarina, Brasil). Após a união foram realizados 3 pontos de oclusão afim de manter a estabilidade da oclusão do paciente e posterior moldagem com utilização de Silicone base leve (Scanlight ${ }^{\circledR}$, Yller, Pelotas, Rio Grande do Sul). A moldagem foi encaminhada ao laboratório de prótese para confecção da barra Metálica (Figura 3F), seguida de prova dos dentes e acrilização da prótese final (Figura 3H e 3I). O paciente recebeu uma prótese total inferior removível após exodontia dos elementos dentários remanescentes.

REVISTA FLUMINENSE DE ODONTOLOGIA - ANO XIV - No 50 - Julho / Dezembro 2018 

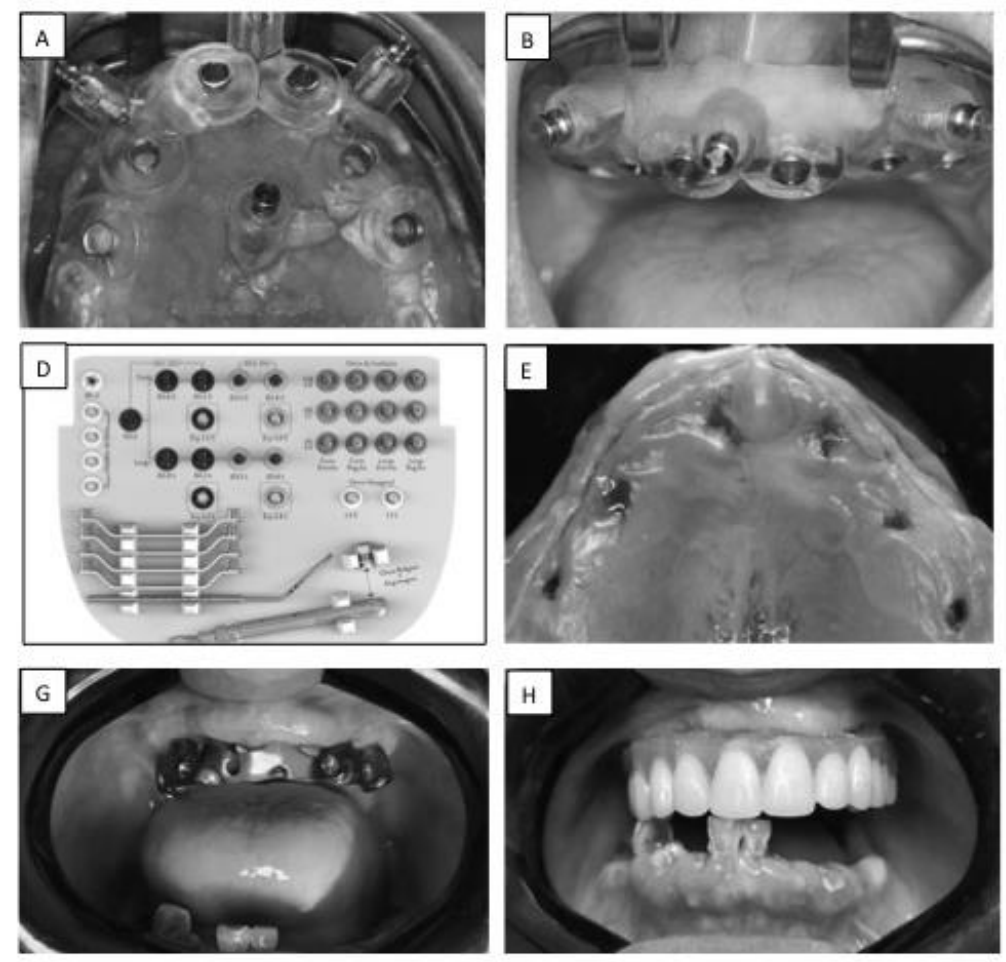
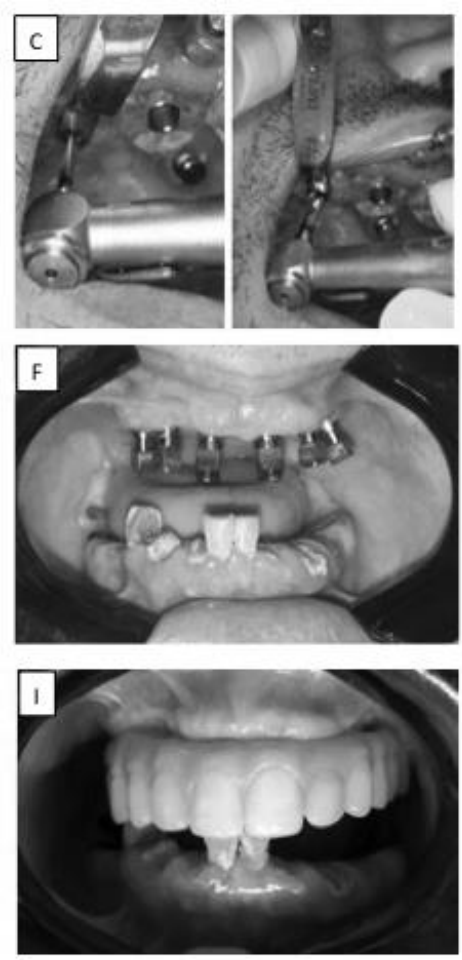

Figura 3. (A e B) Instalação do guia cirúrgico intra-oral; (C) sequência de fresagem para instalação dos implantes; (D) Kit cirúrgico EMFILS® (E)aspecto intra-oral após a instalação dos implantes; (F)posicionamento dos transferentes de moldeira aberta com parafusos de minipilares; (G) Prova da estrutura metálica; (H) prova dos dentes em cera; (I) prótese definitiva instalada

\section{DISCUSSÃO}

O procedimento do tipo All-on-four ou All-on-Six, tem como conceito principal a otimização do procedimento e a reabilitação oral permitindo aos pacientes usarem uma prótese fixa aparafusada sobre quatro ou seis implantes em maxila, mesmo quando a disponibilidade óssea é menor. ${ }^{4}$ No caso apresentado, inicialmente foi eleito o tratamento do tipo All-on-Four por apresentar como vantagem a diminuição do custo total de procedimento assim como maior facilitação da manutenção e higiene das próteses fixas. Porém na confecção do planejamento virtual foi realizado a opção de instalação de mais dois implantes para melhor distribuição biomecânica dos implantes. 
COPYRIGHT @ 2018 INTERNATIONAL JOURNAL OF SCIENCE DENTISTRY | AVAILABLE ONLINE http://www.periodicos.uff.br/index

Relatos na literatura tem mostrado boa precisão da técnica guiada por computador em comparação com uma abordagem analógica. O desvio médio dos implantes inseridos utilizando técnicas de cirurgia guiada é de 1,09 mm na porção mais cervical do implante e de 1,28 mm no ápice e um erro na angulação media de 3,9. Já outros pesquisadores apontam uma variação média mais alta, de 1,45 mm na porção mais cervical do implante e desvio médio de 2,99 $\mathrm{mm}$ no ápice com erro na angulação média de 7,25ㅇ (D'HAESE, J., 2012).

O planejamento virtual pode ser utilizado com indicação, respeitando os critérios de precisão para obter um tratamento de sucesso. No entanto, a variação nos resultados publicados pode estar relacionada a fatores relatados, como guias apoiadas diretamente no tecido gengivalou falta de fixação da guia (D'HAESE, J., 2012).

\section{CONCLUSÃO}

Os protocolos "all-on-four" ou "all-on-six" para reabilitação de maxilares edêntulos através de guia cirurgia computadorizada sem presença de retalho apresenta-se como uma forma eficaz e previsível com maior aceitação por parte do paciente. Porém essa técnica necessita de uma curva de aprendizado por parte do implantodontista.

\section{REFERÊNCIAS BIBLIOGRÁFICAS:}

1- Branemark PI, Adell R, Breine U, Hansson BO, Lindstrom J, Ohlsson A. Intraosseous anchorage of dental prostheses: I. Experimental studies. Scand J PlastReconstrSurg. 1969;3:81-100.

2- Puig CP. 'All-on-four' and 'all-on-six' immediate function with flapless surgery. Eur J Oral Implantol 2010;3(2):155-163.

3- Vieira RA, Melo ACM, Budel LA, Gama JC, de MattiasSartori IA, Thomé G. Benefits of rehabilitation with implants in masticatory function: is patient perception of change in accordance with the real improvement? J Oral Implantol. 2014;40(3): 263-269.

4- Maló $\mathrm{P}$, Lopes A. The rehabilitation of completely edentulous maxillae with different degrees of resorption with four or more immediately loaded implants: a

REVISTA FLUMINENSE DE ODONTOLOGIA - ANO XIV - No 50 - Julho / Dezembro 2018 
COPYRIGHT @ 2018 INTERNATIONAL JOURNAL OF SCIENCE DENTISTRY | AVAILABLE ONLINE http://www.periodicos.uff.br/index

5-year retrospective study and a new classification. Eur J Oral Implantol. 2011;4(3): 227-243.

5- Malo P, Nobre A, Lopes A. The use of computer-guided flaplessimplant surgery and four implantsplaced in immediate function to supporta fixed denture: Preliminary results after a mean follow-up period of thirteen months. J Prosthet Dent 2007; 97: S26-S34.

6- Mourao C, Machado R. "The Reverse Planning: Stages in Total Edentulous Patients for Implant Dentistry". EC Dental Science. 2017;14:172-173.

7- Van Assche N, Vercruyssen M, Coucke W, Teughels W, Jacobs R, Quirynen M. Accuracy of computer- aided implant placement. Clin Oral Implants Res. 2012;23.s6:112-123.

8- D'haese J, Van De Velde T, Elaut L, De Bruyn H. A prospective study on the accuracy of mucosally supported stereolithographic surgical guides in fully edentulous maxillae. ClinImplantDentRelat Res. 2012;14(2): 293-303. 